\title{
Saberes de gestantes com HIV sobre o autocuidado
}

\author{
Knowledge of pregnant women with HIV about self-care \\ Conocimientos de las mujeres embarazadas con HIV en el autocuidado
}

Jeane Rodrigues Miranda Serrão ${ }^{1 *}$, Ivonete Vieira Pereira Peixoto ${ }^{2}$, Camilla Cristina Lisboa do Nascimento$^{2}$, Analu Miranda Serrão², Monica Custodia Abreu Pamplona².

\section{RESUMO}

Objetivo: Conhecer os saberes sobre autocuidado de gestantes com soropositividade para HIV. Métodos: Estudo qualitativo, ocorrido através de um grupo focal realizado com gestantes que apresentam soropositividade para HIV, em um Centro de Referência em HIV/AIDS da rede pública de saúde, situado em Belém-PA. A coleta de dados foi realizada no mês de julho de 2019. Todo o conteúdo do grupo focal foi gravado, transcrito em sua integra, organizado e analisado por meio da utilização de nuvem de palavras, pelo o software IRAMUTEQ. Após isso, foi realizada a interpretação dos os sentidos das palavras nos discursos das gestantes. Resultados: Participaram do grupo 10 gestantes, onde a maioria apresentou saberes sobre o autocuidado, no entendo apresentaram dúvidas em como realizar as práticas dos mesmos. Também foi observado pela totalidade da roda o medo do preconceito que é gerado em torno da temática. Conclusão: Espera-se que este estudo possa auxiliar outras pesquisas e as equipes de saúde para que desenvolvam estratégias que possam sensibilizar a população sobre a necessidade de conhecer e compreender sobre a perspectiva da gestante com HIV.

Palavras-chave: Gestante, Soropositividade para HIV, Autocuidado.

\begin{abstract}
Objective: To know the knowledge about self-care of pregnant women with HIV seropositivity. Methods: Qualitative study, occurred through a focus group conducted with pregnant women who present HIV seropositivity, in a Reference Center in HIV/AIDS of the public health network, located in Belém-PA. Data were collected in July 2019. All the contents of the focus group were recorded, transcribed in its integration, organized and analyzed through the use of word cloud, by the IRAMUTEQ software. After this, the interpretation of the meanings of words was performed in the discourses of pregnant women. Results: 10 pregnant women participated in the group, where the majority presented knowledge about self-care, in the study presented doubts about how to perform their practices. The whole of the wheel was also observed the fear of prejudice that is generated around the theme. Conclusion: It is hoped that this study can help other research and health teams to develop strategies that can sensitize the population about the need to know and understand about the perspective of pregnant women with HIV.
\end{abstract}

Keywords: Pregnant woman, HIV Seropositivity, Self-care.

\section{RESUMEN}

Objetivo: Saber el conocimiento sobre el autocuidado de las mujeres embarazadas con seropositividad del VIH. Métodos: Estudio cualitativo, realizado a través de un grupo focal realizado con mujeres embarazadas que presentan seropositividad con VIH, en un Centro de Referencia en VIH/SIDA de la red de salud pública, ubicado en Belém-PA. Los datos se recopilaron en julio de 2019. Todos los contenidos del grupo de enfoque fueron grabados, transcritos en su integración, organizados y analizados a través del uso de word cloud, por el software IRAMUTEQ. Después de esto, la interpretación de los significados de las palabras se realizó en los discursos de las mujeres embarazadas. Resultados: 10 mujeres embarazadas participaron en el grupo,

\footnotetext{
1 Universidade do Estado do Pará (UEPA), Belém-PA. *E-mail: serraojeanemiranda1@gmail.com
}

¿Universidade do Estado do Pará (UEPA), Belém-PA. 
donde la mayoría presentó conocimientos sobre el autocuidado, en el estudio presentaron dudas sobre cómo llevar a cabo sus prácticas. La totalidad de la rueda también se observó el miedo a los prejuicios que se generan alrededor del tema. Conclusión: Se espera que este estudio pueda ayudar a otros equipos de investigación y salud a desarrollar estrategias que puedan sensibilizar a la población sobre la necesidad de conocer y comprender la perspectiva de las mujeres embarazadas con $\mathrm{VIH}$.

Palabras clave: Mujeres Embarazadas, Seropositividad del VIH, Autocuidado.

\section{INTRODUÇÃO}

A gestante soropositiva é um ser de direitos e de necessidades diferenciadas que se encontra em condições de normatividade alterada. É um ser humano integral, uma síntese de múltiplas relações em seu contexto de vida, de modo que suas necessidades não são somente de ordem prescritiva, mas também de ordem simbólica, material e fisiológica, a exemplo o apoio psicossocial para este grupo (COSTA JJ, et al., 2016).

Estudos apontam para uma sobrecarga emocional das mulheres que vivem com o Vírus da Imunodeficiência Humana (HIV), sendo esse período permeado por medos, culpas e preconceitos. As principais preocupações apresentadas pelas mulheres após terem acesso às informações dos possíveis riscos, neste período, referem-se à saúde do filho, quanto à possibilidade de infecção da criança, frustração diante da expectativa da não amamentação e a preocupação com o parceiro e a família. Portanto, cada mulher lidará de forma singular com a gestação, de acordo com fatores individuais, familiares, históricos e socioculturais (LEVANDOWSKI DC, et al., 2014).

Ressalta-se que os sentimentos e os desejos desenvolvidos durante a gestação, incluindo as expectativas e planos após o parto, podem influenciar os próximos 12 meses da criança, principalmente em seu vínculo com a mãe (SABROZA AR,et al., 2011).

Bringe IAPV et al. (2015) destaca que a equipe multiprofissional, no acompanhamento das gestantes soropositivas, almeja com suas ações fortalecer o vínculo com a paciente, e promover um cuidado humanizado, integral e ético. Haja vista que o vínculo e a confiança depositadas pela paciente no profissional de saúde farão a diferença em relação, à compreensão da doença e a adesão ao tratamento, viabilizando o enfrentamento positivo, proporcionando maior aceitação e tranquilidade por parte da mulher.

No contexto de gravidez as mulheres precisam tomar decisões, por exemplo, o fato de não poder amamentar, o uso das medicações, entre outros assuntos que afetam a vida desta gestante e repercutem no contexto familiar. Desta forma, a equipe de saúde pode intervir nesse contexto, dando-lhes o apoio necessário para o enfrentamento da doença, como a presença da escuta qualificada nos atendimentos prestados no serviço. Evidencia-se, então, a importância da dimensão que é conferida a relação profissional-cliente como participantes na busca da promoção da saúde numa realidade em construção de relações, saúde, vida. Assim, a assistência a esse grupo requer sensibilização, ética e capacidade de trabalho em grupo que possam fomentar decisões responsáveis nessas esferas delicadas de cuidados (BRINGEL APV, et al., 2015).

A gravidez compõe um cenário real e diário do assistir na atenção básica e precisa ser trabalhada na perspectiva da integralidade das ações para serem construídas ferramentas que se apresente como formas DE promoção do autocuidado (VANESSA KSL, et al. 2019). Diante disso, é necessário identificar os saberes dessas mulheres a respeito do seu cuidado para a construção dessas estratégias dentro da atenção básica.

Com isto, o objeto desse estudo é conhecer os saberes sobre o autocuidado de gestantes com soropositividade para HIV.

\section{MÉTODOS}

Realizou-se um estudo descritivo, de natureza qualitativa, do tipo pesquisa-participante. O cenário foi o centro de referência em HIV da rede pública de saúde, situado em Belém-PA. 
Os dados foram produzidos por meio da técnica de Grupo Focal, que se caracteriza por uma sessão grupal, de pessoas organizadas de forma intencional. Utilizou-se esta técnica por sua aplicação em pesquisas que se propõem a entender atitudes e comportamentos das pessoas no ambiente organizacional, possibilitando a investigação de como pensam/percebem determinado tema, fato ou acontecimento em contato direto com eles, explorando as necessidades e identificando atitudes, sobretudo mediante a perspectiva de pesquisa qualitativa (MORGAN C, 2010).

Foi realizado um encontro com o grupo para a realização da conversação. Este encontro aconteceu na própria unidade no mês de julho de 2019 , conforme a disponibilidade das participantes e pactuação com a enfermeira $\mathrm{E} A$ gestora da Unidade. $O$ convite às participantes foi realizado durante a sua consulta rotineira de pré-natal, explicando-se os objetivos do estudo e importância de sua participação. A partir de sua anuência organizou-se o melhor dia para o encontro.

Este teve duração em média de duas horas, atendendo às sugestões para realização da técnica, para que a produção dos dados fosse funcional, evitando o cansaço dos participantes e a manutenção do foco no tema, propiciando informações suficientes para uma boa análise (SILVA MG, et al., 2013). Ressalta-se que foi utilizado como instrumento de organização do grupo um roteiro-guia, elaborado pelas pesquisadoras, com indagações sobre os saberes das gestantes sobre o autocuidado e sobre o formato e conteúdo da tecnologia.

Ressalta-se que compuseram o grupo focal, além das participantes, a moderadora do grupo e cinco observadores, sendo estes acadêmicos do curso de graduação em enfermagem, que realizaram as gravações das falas e registro das discussões em um diário de campo. Destaca-se que, no começo da realização do grupo focal, foram enfatizados os objetivos e a importância da realização do encontro, deixando-se claro que todos os relatos fossem de grande valia para o grupo focal e que não havia certo ou errado nas opiniões emitidas, pois a discussão era totalmente aberta em torno do tema proposto e qualquer tipo de reflexão e contribuição era importante para a pesquisa.

Todo o conteúdo do estudo foi organizado e analisado por meio da utilização de nuvem de palavras, pelo - software Interface de $R$ pour les Analyses Multidimensionnelles de Textes et de Questionnaires (IRAMUTEQ). Dessa forma, as palavras foram agrupadas e organizadas graficamente de acordo com a sua frequência, o que proporcionou facilmente a sua identificação, a partir de um único arquivo, denominado corpus, que reúne os textos originados pelos relatos durante o grupo focal (CAMARGO BV e JUSTO AM, 2013).

Assim, cada resposta caracterizou um texto, e o conjunto desses textos constituiu o corpus de análise desta pesquisa, o qual após as etapas de processamento foram interpretados os sentidos das palavras nos discursos das gestantes.

A pesquisa obedeceu aos preceitos éticos da Resolução n 466/2012 do Conselho Nacional de Saúde (BRASIL, 2013). Foi aprovado no Comitê de Ética em Pesquisa da Universidade do Estado do Pará, CAAEE: 13015219.6.0000.5174.

A todas as participantes foi apresentado o Termo de Consentimento Livre e Esclarecido para conhecerem os objetivos do estudo e manifestarem livremente seu interesse em participar. Para preservar suas identidades, as mesmas foram identificadas com nomes de flores.

\section{RESULTADOS E DISCUSSÃO}

Os resultados serão apresentados em dois aspectos: caracterização das gestantes participantes e análise lexical - método nuvem de palavras.

\section{Participantes do estudo}

Participaram da pesquisa 10 gestantes portadoras do vírus HIV, em média de idade de 22 a 26 anos. Como observado na tabela 1, a maioria (50\%) encontra-se em união estável, seguido de $30 \%$ casadas e $20 \%$ solteiras. 
Tabela 1 - Perfil social das gestantes participantes da pesquisa. Belém-PA, 2019.

\begin{tabular}{|c|c|c|c|c|c|c|}
\hline GESTANTE & IDADE & ESCOLARIDADE & OCUPAÇÃO & $\begin{array}{c}\text { ESTADO } \\
\text { CIVIL }\end{array}$ & $\begin{array}{c}\text { TEMPO DE } \\
\text { DIAGNÓSTICO }\end{array}$ & RELIGIÃO \\
\hline JASMIM & $\begin{array}{c}18 \text { a } 22 \\
\text { anos }\end{array}$ & $\begin{array}{c}\text { Fundamental } \\
\text { incompleto }\end{array}$ & $\begin{array}{c}\text { Sem } \\
\text { profissão }\end{array}$ & Solteira & 10 meses & Evangélica \\
\hline $\begin{array}{c}\text { MARGARID } \\
A\end{array}$ & $\begin{array}{c}22 \text { a } 26 \\
\text { anos }\end{array}$ & $\begin{array}{c}\text { Fundamental } \\
\text { completo }\end{array}$ & $\begin{array}{l}\text { Sem } \\
\text { profissão }\end{array}$ & Solteira & 3 anos & Católica \\
\hline GIRASSOL & $\begin{array}{c}22 \text { a } 26 \\
\text { anos }\end{array}$ & $\begin{array}{l}\text { Fundamental } \\
\text { incompleto }\end{array}$ & $\begin{array}{l}\text { Sem } \\
\text { profissão }\end{array}$ & Casada & 11 meses & Católica \\
\hline$A Z A L E ́ I A$ & $\begin{array}{c}\text { Acima } \\
\text { de } 36 \\
\text { anos }\end{array}$ & Médio completo & Cozinheira & Casada & 14 anos & Evangélica \\
\hline BEGÔNIA & $\begin{array}{c}30 \text { a } 36 \\
\text { anos }\end{array}$ & Médio completo & $\begin{array}{c}\text { Sem } \\
\text { profissão }\end{array}$ & Casada & 7 anos & Católica \\
\hline $\begin{array}{c}\text { CALÊNDUL } \\
A\end{array}$ & $\begin{array}{c}26 \text { a } 30 \\
\text { anos }\end{array}$ & Médio incompleto & Vendedora & $\begin{array}{l}\text { Relação } \\
\text { estável }\end{array}$ & 2 anos & Evangélica \\
\hline GÉRBERA & $\begin{array}{c}22 \text { a } 26 \\
\text { anos }\end{array}$ & $\begin{array}{l}\text { Fundamental } \\
\text { incompleto }\end{array}$ & $\begin{array}{c}\text { Sem } \\
\text { profissão }\end{array}$ & $\begin{array}{l}\text { Relação } \\
\text { estável }\end{array}$ & 10 meses & Evangélica \\
\hline $\begin{array}{c}\text { HORTÊNSI } \\
A\end{array}$ & $\begin{array}{l}\text { Acima } \\
\text { de } 36 \\
\text { anos }\end{array}$ & Médio completo & $\begin{array}{c}\text { Sem } \\
\text { profissão }\end{array}$ & $\begin{array}{l}\text { Relação } \\
\text { estável }\end{array}$ & 5 anos & Evangélica \\
\hline HIBISCO & $\begin{array}{c}26 \text { a } 30 \\
\text { anos }\end{array}$ & Médio completo & $\begin{array}{c}\text { Auxiliar de } \\
\text { serviços } \\
\text { gerais }\end{array}$ & $\begin{array}{l}\text { Relação } \\
\text { estável }\end{array}$ & 2 anos & Evangélica \\
\hline LÍRIO & $\begin{array}{c}26 \text { a } 30 \\
\text { anos }\end{array}$ & Médio completo & $\begin{array}{c}\text { Sem } \\
\text { profissão }\end{array}$ & $\begin{array}{l}\text { Relação } \\
\text { estável }\end{array}$ & 4 anos & Evangélica \\
\hline
\end{tabular}

Fonte: Serrão JRM, Peixoto IVP, Nascimento CCL, Serrão AM, Pamplona MCA, 2019.

Em relação ao tempo de diagnóstico de HIV, a média de idade foi de 4 anos (mínimo menos de 10 meses e máximo de 14 anos). Quando questionadas sobre o uso de terapia antirretroviral, todas as gestantes afirmaram estar realizando o mesmo de forma regular e correta. O uso da terapia antirretroviral é de suma importância, visto que se trata de uma forma de prevenir a transmissão vertical do HIV e possibilitar a boa qualidade de vida para a gestante (FARIA ER, 2014).

Sobre o nível de escolaridade, $40 \%$ não realizaram o ensino médio (sendo 3 cursaram o ensino fundamental incompleto e 1 o ensino fundamental completo) e $60 \%$ pararam no ensino médio (sendo 1 não concluiu o ensino médio e 5 concluíram). Nenhumas das participantes tinham planos próximos de finalizar os seus estudos ou irem para o ensino superior.

Quando questionadas sobre a forma de trabalho das mesmas, 70\% não apresentavam uma ocupação no momento e $30 \%$ apresentava ocupação que tinham pagamento 1 salário mínimo. Segundo Lima SS et al. (2017), o baixo nível socioeconômico e de escolaridade se apresenta como sendo um dos fatores que influenciam na adesão das gestantes soropositivas desde o aconselhamento até o acompanhamento no prénatal, visto que as gestantes não apresentam condições financeiras para comparecerem as unidades de saúde, além do que, a baixa escolaridade dificulta a busca por informações e impede a compreensão sobre a importância de se realizar o acompanhamento.

Quanto ao histórico obstétrico, o número médio de filhos e o número de gestações anteriores foram, respectivamente, de 2 filhos e 3 gestações contando com a atual. Não houve relato de abortos (espontâneos ou não) ou natimortos. 


\section{Análise dos saberes das gestantes sobre o autocuidado - nuvem de palavras}

Observa-se que as participantes apresentam breve ou pouco conhecimento sobre o significado do que é autocuidado, uma vez que pelo método de nuvem de palavras, que agrupa as palavras e as organiza graficamente em função da sua frequência, a palavra cuidar foi a que teve maior frequência no corpus-12 vezes, como consta na Figura 1.

Figura 1 - Nuvem de palavras. Belém-PA, 2019.

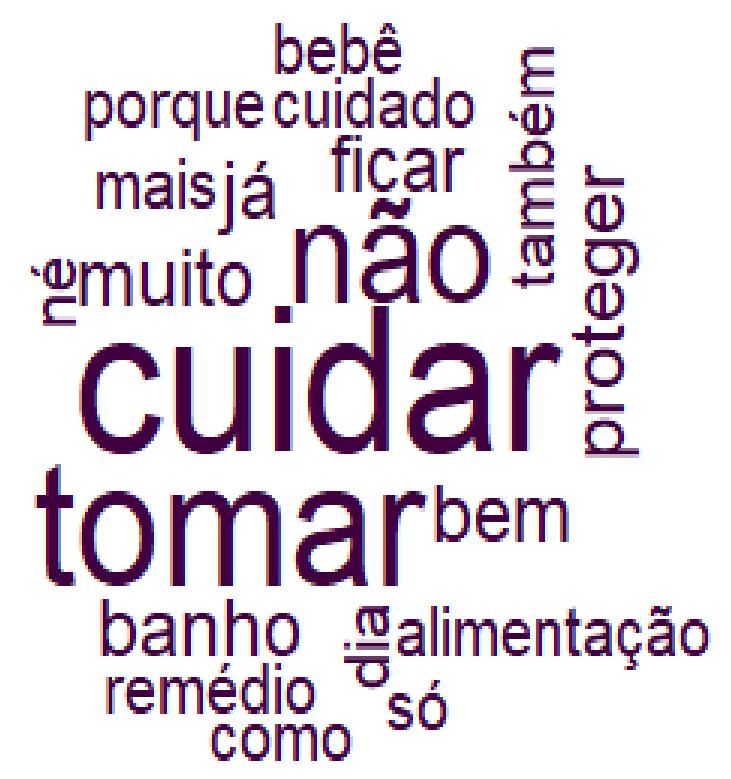

Fonte: Serrão JRM, Peixoto IVP, Nascimento CCL, Serrão AM, Pamplona MCA, 2019.

Observa-se, pela figura acima, que as palavras que se apresentam com maior frequência aparecem em maior destaque do que as outras, que foram posicionadas de forma aleatória no corpus de análise da pesquisa. Com isto, é notório na fala das participantes que a palavra "cuidar", o qual apresentou o conceito de cuidar de si mesma.

"Se cuidar, se proteger, cuidar de mim e do meu filho. Tomar os xaropes por um período sem parar depois que nasce. Alimentação saudável, lazer, cuidar seria proteger, cuidar de mim e do meu filho, fazer exercício físico." (Girassol)

"Se amar, se propiciar coisas boas pra ir se cuidando, ir no shopping, passear, tomar banho no igarapé, tirar um dia para curtir a família, de preferência só com elas, ficar bem." (Calêndula)

"Proteger o bebê e se cuidar também, como tomar o remédio, fazer os exames, precisa ter mais apoio da família, acompanhamento com o psicólogo é importante também. Eu gosto de ficar com grupo dançando forró, só isso já deixa o psicológico bem, não custa nada e esqueço os problemas, isso tudo faz parte de se cuidar." (Lírio)

Com relação ao entendimento das gestantes sobre o que significa o autocuidado, a maioria relatou a importância de uma alimentação balanceada durante o período gestacional, o que é também observado em uma roda de conversa realizada no Centro de Referência da Assistência Social (CRAS) em Acarape - CE, em 2015 (VANESSA KSL, et al. 2019).

Para o alcance do estado nutricional ideal, a gestante necessita de um acompanhamento adequado, com estratégias para a educação alimentar, suprindo, assim, todas as necessidades da mulher e do feto no período gestacional (VANESSA KSL, et al. 2019). 
Destaca-se ainda que as orientações de autocuidado para o período gestacional devem ser iniciadas durante as primeiras consultas de pré-natal e, se possível, ser reafirmada por meio de grupos educativos entre gestantes e também em sala de espera. (SILVA SR, et al., 2014)

Corroborando com tal afirmativa, segundo Scherer LM (2009), as trocas de experiências que possam ocorrer em rodas de conversas e entrevistas com mulheres grávidas se tornam oportuna à incorporação de medidas de autocuidado. Quando discutido sobre quais informações sobre cuidado que as mesmas devem apresentar durante a sua gestação, evidenciou-se que as participantes reconhecem que o cuidado com elas próprias deve ser amplamente maximizado, porém todas apresentam dúvidas nos cuidados que devem ser realizados durante o período gestacional e puerpério.

"Ainda sei muito pouco, mas o pouco que sei foi aqui pelo pré-natal, quando descobri [a gestação] nem me falaram nada como ia sei ser." (Azaléia)

"Ainda tô descobrindo leio um pouco, pergunto, me falaram quando comecei aqui mas ainda tenho muitas dúvidas." (Begônia)

Com isso, pontua-se a necessidade, ao decorrer do período gestacional, QUE a mulher receba orientações e encorajamento relacionados a busca por medidas de prevenção de intercorrências no crescimento e no desenvolvimento do bebê (VANESSA KSL, et al. 2019).

Reflete-se com isso que o autocuidado torna-se benefício a todas as gestantes, em destaque aquelas com soropositividade para HIV, visto que com isto as mesmas entendem a importância de satisfazer as necessidades do corpo e da mente, com consequência a melhora do estilo de vida, a negação para hábitos nocivos, o desenvolvimento de uma alimentação sadia, o empoderamento sobre os fatores de risco que levam às doenças e adoção medidas de prevenção de doenças (LIMA SS, et al., 2017). Afirmando o que se apresentou no grupo focal quando o indagadas sobre quais os cuidados que as mesmas realizam atualmente durante a gestação, como observado nos relatos abaixo.

"Importante fazer os exames e ver se está tudo bem para o parto e o bebê." (Jasmin)

"Se cuidar, tomar banho, cuidar da higiene, ficar bem consigo mesmo." (Gérbera)

"Tenho que ter a consciência que tenho que estar bem para o meu bebê ficar saudável e bem. Preciso ficar bem não só no aspecto físico, mas na saúde, no psicológico." (Margarida)

Estudos de Rubiano MYL (2016) destaca que o desejo das gestantes de terem os seus filhos sem o vírus e que elas possam criá-los e educá-los, fazem com que as mesmas tenham um maior incentivo de realizar com eficácia o tratamento para o HIV. Isto é, o principal motivo para a manutenção e para a realização correta do tratamento durante a gestação é que elas passam a perceber o filho como extensão de si próprio e, com isso, necessitarão estar saudáveis para realizar os cuidados necessários relacionados à maternidade.

Ressalta-se que a maioria das gestantes apresentou vínculo de confiabilidade com os profissionais de saúde atuante nas consultas de pré-natal, o que corrobora com pesquisas de Hernandes CP (2019). No entanto, todas mostraram que apresentam também outros meios de conseguir informações sobre como realizar os cuidados durante da gestação e que se sentem mais confortáveis por esses.

"Pelos profissionais, um pouco na internet, mas deixa a gente muito confusa." (Margarida)

"Eu gosto muito de perguntar, pros médicos e enfermeiros, as pessoas que me atendem. Quando a gente vai buscar o remédio acaba conhecendo outras pessoas e vai rolando uma amizade, [...] aí conversamos e vamos aprendendo ainda mais uns com os outros, [...] perguntando e conversando de preferência para quem entende e vive isso, tenho uma amiga que teve primeiro que eu e ela me ensinou muito, me passou tudinho, coisas que nem na internet tem e ainda sentimos ruim quando lemos." (Hibisco) 
Durante o grupo, foi possível identificar que público apresentava, de forma geral, poucas informações acerca do autocuidado e abordagem em geral. Além disso, foi notória a valorização e atenção das falas das multíparas acerca do assunto pelas primigestas. O que também foi identificado em estudos de Silva SR et al. (2014), que aborda que a ausência ou ineficiência de orientações por parte dos profissionais sobre os cuidados no período gestacional, levam as mulheres a buscarem informações em outras fontes como família, amigos, televisão e revistas. Entretanto, tais orientações podem ser repassadas de formas errôneas, podendo influenciar nas práticas de autocuidado e prejudicar a qualidade de vida da gestante.

Observa-se então a importância do vínculo entre os profissionais de saúde e das gestantes para o esclarecimento de dúvidas, para com isso se apresente maximização eficazes dos conhecimentos das grávidas e resolução de dúvidas corriqueiras em tal período (SILVA SR, et al., 2014). Além disso, as gestantes apresentaram preocupação sobre os preconceitos em torno das pessoas que apresentam soropositividade para HIV e como tal assunto pode influenciar nos cuidados com o filho.

"Eu tenho que estar bem forte, ele [filho] quando nascer vai frequentar os mesmos locais que eu, muitas vezes vai me questionar e perguntar algumas coisas e eu tenho que estar preparada para auxiliar ele e fazer ele compreender e de sentir seguro, não se sentir discriminado por mim." (Margarida)

Ressalta-se que o preconceito é um dos aspectos principais que influenciam de forma negativa o processo de aceitação e cuidados relacionado à temática. Ele foi pontuado como forma de preocupação por todas as gestantes, que demonstraram apresentar medo de sofrer situações de discriminação. Hernandes CP (2019) aponta que tal preconceito desde as ações em saúde destinadas a portadores da doença e essa totalidade dificulta o enfrentamento da doença, muitas vezes, culminando em pouco acolhimento e transmissão de informações deficitárias no pré-natal.

Outro estudo realizado com mulheres gestantes soropositivas para HIV destacam os problemas na aceitação da gestação, pois as mesmas não planejavam engravidar pela sua condição clínica e também por não apresentarem condição financeira que permitissem a continuidade da gestação e, por isso, até pensavam em abortar (DARMONT MQR, et al., 2011).

É notório então pelos relatos e pelos estudos de Leal AF et al. (2012), que o acesso ao atendimento multidisciplinar é uma forma eficaz de auxiliar no processo de aceitação. Isto é, o acompanhamento interdisciplinar é primordial para tais gestantes no âmbito biopsicossocial.

Portanto, observa-se que o enfrentamento da doença, a realização de medidas profiláticas e o apoio recebido de familiares e da sociedade levam as gestantes soropositivas a ter controle de sua vida e consequentemente, aumentam sua expectativa e qualidade de vida (FARIA ER, 2014).

\section{CONSIDERAÇÕES FINAIS}

Diante do exposto, é notória a importância do processo de autocuidado das gestantes, principalmente das com HIV positivo. Ressalta-se ainda que a política de saúde voltada para esse grupo populacional deve incentivar o processo de aceitação e proporcionar sensibilização de formas de cuidados de maneira eficaz. Espera-se que este estudo possa auxiliar outras pesquisas e as equipes de saúde para que desenvolvam estratégias que possam sensibilizar a população sobre a necessidade de realizar acompanhamento da gestante.

\section{REFERÊNCIAS}

1. BRINGEL APV, et al. Vivência de gestantes com HIV. Cienc Cuid Saude, 2015; 14(2): 1043-1050.

2. CAMARGO BV, JUSTO AM. IRAMUTEQ: um software gratuito para análise de dados textuais. Temas psicol, 2013; 21(2):513- 18.

3. COSTA JJ, et al. Questões éticas implicadas no cuidado a gestantes portadoras de HIV. Revista Brasileira de Tecnologias Sociais, 2013; 3(1): 41-52. 
4. DARMONT MQR, et al. Adesão ao pré-natal de mulheres HIV+ que não fizeram profilaxia da transmissão vertical: um estudo sócio-comportamental e de acesso ao sistema de saúde. Cad. Saúde Pública. 2010;26(9): $1788-96$.

5. FARIA ER. Gestação e HIV: preditores da adesão ao tratamento no contexto do pré-natal. Psic: Teor Pesq. 2014; 30(2): 197-203.

6. LEAL AF, et al. Medidas de prevención de la transmisión vertical del VIH empleadas por madres de niños seropositivos. Invest Educ Enferm. 2012; 30(1): 44-54.

7. LEVANDOWSKI DC, et al. Maternidade e HIV: revisão da literatura brasileira (2000-2014). Arquivos Brasileiros de Psicologia, 2017; 69(2): 34-51.

8. LIMA SS, et al. HIV na gestação: pré-natal, parto e puerpério. Ciência \& Saúde, 2017; 10(1): 56-61.

9. LIMA VKS, et al. Educação em saúde para gestantes: a busca pelo empoderamento materno no ciclo gravídicopuerperal. Rev Fun Care Online. 2019; 11(4):968-975.

10. MORGAN C. Using the focus group in marketing research. 2010. Disponível em: \&lt;http://www.b2binternational.com/library/whitepapers/whitepapers08.php\&gt. Acesso em: 16 ago. 2019.

11. RUBIANO YLM, et al. Más allá de mí: cuidarme para proteger mi hijo. Experiencias de mujeres gestantes con VIH/SIDA. Rev Univ Ind Santander Salud, 2016; 48(3): 353-363.

12. SABROZA AR, et al. Algumas repercussões emocionais negativas da gravidez precoce em adolescentes do município do Rio de Janeiro (1999-2001). Cad. Saúde Pública 20, 2011; 1(1): 130-137.

13. SCHERER LM, et al. Gestantes/puérperas com HIV/AIDS. Esc Anna Nery Rev Enferm, 2009; 13 (2): $359-65$.

14. SILVA MG, et al. Publicações que utilizaram o grupo focal como técnica de pesquisa: o que elas nos ensinam? Ciênc Cuid Saúde, 2013; 12(2): 398-406.

15. SILVA SR, et al. Práticas de autocuidado desenvolvidas por gestantes atendidas em um ambulatório de pré-natal. Rev. Eletr. Enf., 2014; 16(4):812-21.

16. VANESSA KSL, et al. Educação em saúde para gestantes. J. res.: fundam. care. Online, 2019; 11(4): $968-975$. 